\title{
Erratum: Linear Boltzmann transport for jet propagation in the quark-gluon plasma: Elastic processes and medium recoil [Phys. Rev. C 91, 054908 (2015)]
}

Yayun He, Tan Luo, Xin-Nian Wang, and Yan Zhu

(Received 12 December 2017; published 11 January 2018)

DOI: 10.1103/PhysRevC.97.019902

After the publication of this article, we found several typographical errors in the original paper involving the degeneracy and other factors in the Boltzmann equations and scattering rates. These are just typographical errors in the paper. The correct factors are used in the actual Monte Carlo code of the linear Boltzmann transport model.

An overall factor $g_{b} / 2$ is missing on the right-hand side of Eq. (1). The correct form should be

$$
\begin{aligned}
p_{1} \partial f_{a}\left(p_{1}\right)= & -\int \frac{d^{3} p_{2}}{(2 \pi)^{3} 2 E_{2}} \int \frac{d^{3} p_{3}}{(2 \pi)^{3} 2 E_{3}} \int \frac{d^{3} p_{4}}{(2 \pi)^{3} 2 E_{4}} \\
& \times \sum_{b(c, d)} \frac{g_{b}}{2}\left[f_{a}\left(p_{1}\right) f_{b}\left(p_{2}\right)-f_{c}\left(p_{3}\right) f_{d}\left(p_{4}\right)\right]\left|M_{a b \rightarrow c d}\right|^{2} S_{2}(s, t, u)(2 \pi)^{4} \delta^{4}\left(p_{1}+p_{2}-p_{3}-p_{4}\right),
\end{aligned}
$$

where $g_{b}$ is the degeneracy factor for medium parton $b$ in the Boltzmann equation. The values of $g_{g}$ and $g_{q}$ are given in the text following Eq. (1).

In Eq. (4), the degeneracy factor $g_{b}$ is missing on the right-hand side of the equation for the scattering rate. The correct equation should be as follows:

$$
\Gamma_{a b \rightarrow c d}=\frac{g_{b}}{2 E_{1}} \int \frac{d^{3} p_{2}}{(2 \pi)^{3} 2 E_{2}} \int \frac{d^{3} p_{3}}{(2 \pi)^{3} 2 E_{3}} \int \frac{d^{3} p_{4}}{(2 \pi)^{3} 2 E_{4}} f_{b}\left(p_{2}\right)|M|_{a b \rightarrow c d}^{2}(s, t, u) S_{2}(s, t, u)(2 \pi)^{4} \delta^{(4)}\left(p_{1}+p_{2}-p_{3}-p_{4}\right) .
$$

Similarly in Eq. (9), the degeneracy factor $g_{b}$ is missing on the right-hand side of the equation for the differential scattering rate. The correct equation should be as follows:

$$
\Gamma_{a b \rightarrow c d}=\frac{g_{b}}{16 E_{1}(2 \pi)^{4}} \int d \theta_{2} \int d \theta_{3} \int d \phi_{23} \int d E_{3} f_{b}\left(E_{2}, T\right)|M|_{a b \rightarrow c d}^{2}(s, t, u) S_{2}(s, t, u) \frac{E_{2} E_{3} \sin \theta_{2} \sin \theta_{3}}{E_{1}\left(1-\cos \theta_{12}\right)-E_{3}\left(1-\cos \theta_{23}\right)} .
$$

The results and conclusions of the original article are not affected. 